\title{
Können wir denn noch umdenken?
}

A ufforderungen zum Umdenken in der Medizin gibt es, seitdem es die Medizin gibt. Galen, Harvey oder Barnard verwarfen substanzielle Konzepte. Andere bedauerten, dass der Arzt gezwungen wird, die Einmaligkeit von Therapiekonzepten preiszugeben - dies beklagt auch Lown in seinem Buch „Die verlorene Kunst des Heilens - Anleitung zum Umdenken“. Lown sah sein Werk allerdings vor dem Hintergrund, medizinische Prinzipien einer marktorientierten Medizin zu opfern. Die Politik und ihre bürokratische Exekutive würden ermächtigt, ein profitorientiertes Gesundheitswesen auf Kosten einer wahren Fürsorge für die Kranken zu schaffen.

Auch Kollege Jörg-Dietrich Hoppe warnte auf dem 107. Deutschen Ärztetag im Mai 2004 in Bremen vor einer völligen Kommerzialisierung des Gesundheitswesens, und davor, dass es statt eines Wettbewerbes um Qualität einen Wettstreit um Profite geben werde.

Schließlich monierte unser ehemaliger Bundespräsident Johannes Rau - ebenfalls vor dem Deutschen Ärztetag 2004 -, dass wir uns alle daran gewöhnt haben, vom Patienten als „Kunden“ zu reden. Rau dachte um: „Ärzte sind keine Anbieter, Patienten sind keine Kunden. Gesundheitsbewusstsein ist gut, aber es ist keine Ware.“ Recht hat er! Schade, dass diese Aufforderung noch keinen Ruck bewirkt hat. Dagegen treiben die DRGs, Fallpauschalen und vieles mehr zu einer zeitlichen und technischen Perfektionierung - gelegentlich auch ohne sichere Indikationsstellung.

Ich will dabei nicht einmal von einer Profitindikation sprechen. Nur besteht die Gefahr, dass die Kunst des Zuhörens, des Beratens und des körperlichen Untersuchens als diagnostische Methoden bald der Vergangenheit angehören, genauso wie auch konservative Behandlungsformen. Im vorliegenden Heft des klinikarzt finden Sie noch - oder besser wieder - solche Verfahren, die den ganzen Arzt als Person verlangen und versuchen, die Heilkräfte des Patienten, hier am Beispiel der Wundheilung, zu stärken. Und das neben moderner operativer Technik, ohne die es in der Medizin nicht funktioniert. Allerdings geht es auch ohne die Triebfeder des Profits.
Hoffen wir, dass unsere medizinische Versorgung in der gegenwärtigen Landschaft nicht nur „flächendeckend“ bleibt. Dies ist im Übrigen ein ebenso unschöner Ausdruck wie die Bezeichnung unserer Patienten als Kunden. „Menschendeckend“ klingt zwar nicht besser, wäre aber zutreffender. Hoffentlich wird es uns im gegenwärtigen heillosen Getümmel der Reformen gelingen, das wirtschaftlich veränderte Gesundheitswesen mit einem menschlichen Gesicht zu versehen.

Wir müssen in der Medizin erneut den Menschen, das Humanum, in den Fordergrund stellen. Hierfür sind wir bereit, ständig umzudenken ... nachzudenken.

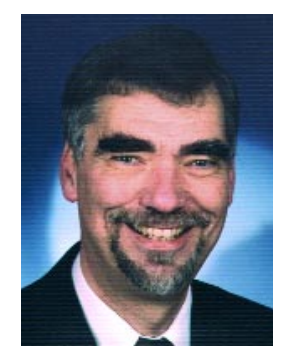

Prof. Dr. W. Hardinghaus, Ostercappeln

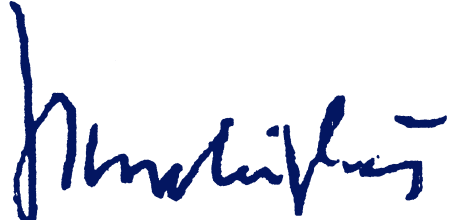

